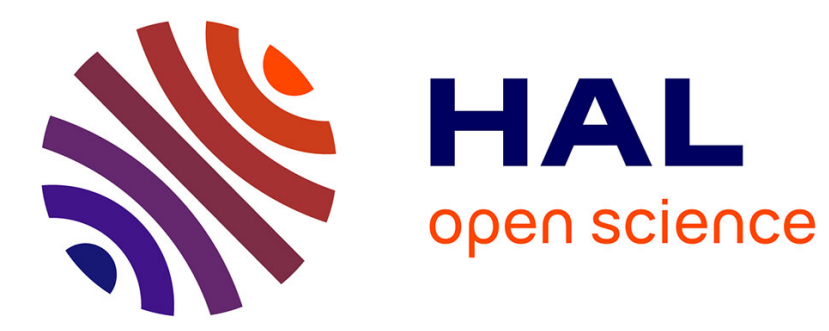

\title{
Simultaneous Sensing and Actuating Compliance-Adaptive Apparatus for Upper Limb Rehabilitation
}

\author{
Hossein Mosuavi Hondori, Shi Hao Shen, Shih-Fu Ling
}

\section{To cite this version:}

Hossein Mosuavi Hondori, Shi Hao Shen, Shih-Fu Ling. Simultaneous Sensing and Actuating Compliance-Adaptive Apparatus for Upper Limb Rehabilitation. RSI/ISM International Conference on Robotics and Mechatronics, Feb 2013, Iran. hal-00797201

\section{HAL Id: hal-00797201 \\ https://hal.science/hal-00797201}

Submitted on 6 Mar 2013

HAL is a multi-disciplinary open access archive for the deposit and dissemination of scientific research documents, whether they are published or not. The documents may come from teaching and research institutions in France or abroad, or from public or private research centers.
L'archive ouverte pluridisciplinaire HAL, est destinée au dépôt et à la diffusion de documents scientifiques de niveau recherche, publiés ou non, émanant des établissements d'enseignement et de recherche français ou étrangers, des laboratoires publics ou privés. 


\section{Simultaneous Sensing and Actuating Compliance- Adaptive Apparatus for Upper Limb Rehabilitation}

\author{
Hossein Mousavi Hondori \\ Department of Mechanical and \\ Aerospace Engineering \\ University of California, Irvine \\ USA \\ hoss0001@e.ntu.edu.sg
}

\author{
Shen Shi Hao \\ School of Mechanical and Aerospace \\ Engineering \\ Nanyang Technological University \\ Singapore \\ shen0013@e.ntu.edu.sg
}

\author{
Ling Shih-Fu \\ School of Mechanical and Aerospace \\ Engineering \\ Nanyang Technological University \\ Singapore \\ msfling@ntu.edu.sg
}

\begin{abstract}
Full or partial loss of functionality of the upper limbs is usually associated with strokes, spinal cord injury and many other similar injuries. Traditional rehabilitation treatment however is extremely labor intensive and are usually only available at hospital. However a lot of studies have shown that through the use of repetitive robotic practice can help the patients to recover. In this paper, a 4-bar linkages mechanism is designed and constructed as the upper limb rehabilitation apparatus which provided a set of motions for upper limb rehabilitation and a sensor-less method of assessing the patient arm using motor. The concept of using the input electrical impedance for monitoring the output mechanical impedance is then experimentally tested and justified in experiments.
\end{abstract}

Index Terms-Simultaneous sensing cum actuating, de motor, electrical impedance, mechanical impedance, robotic rehabilitation, 4-bar mechanism.

\section{INTRODUCTION}

Robotics is well established as a key solution to labor intensive tasks among which physical therapy (PT) and rehabilitation is a repetitive, labor intensive task which is not an exception. The use of robotics in this field have emerged into a new field of research called "Robotic Rehabilitation" (RR). Prior to RR training and assessment of human motor function had remained a challenge for years; this is because of the complexity of human brain and the subjective nature of the assessment. Subjective methods of assessment such as FuglMeyer [1] were and are still used; they are generally qualitative questionnaires to score the performance of subjects. For clinical purposes, though, these methods are still widely used because of their simplicity. Researchers recently tried to use robots for the assessment besides the physical therapy [2]. Their robots were basically designed for performing physical therapy exercises and because of the capabilities of a robot they tried to use the same robot for assessment purposes. Having measured forces with respect to displacement, they measured stiffness of the arm. However, their method was not a direct, real time, and in-situation measurement of the stiffness of the arm. On the other hand, a robot might be very complicated and expensive; setup and maintenance of it can be very difficult as well. Hence, there still remains a room for a more reliable, convenient, and efficient tool for the assessment of motor function. Furthermore, what the robot measures is also a very important issue for the assessment.

Hogan [3] and Hondori [4] showed that the value of mechanical impedance for the upper arm, which is set at the elbow joint, is very important. They examined the postulate that antagonist muscle's co-activation is to generate mechanical impedance and therefore this is necessary to perform some tasks. A typical case with necessity of the antagonist activation is performing a dynamically unstable task. Burdet [5] showed that human learns to stabilize unstable dynamics by optimizing mechanical impedance. Darainy [6] has reported that the EMG patterns of dynamic learning reveals a considerable portion of co-activation in mechanically stable tasks. Therefore, this is not only in case of unstable dynamics that the CNS co-contracts the antagonists to control the impedance of the limb, but also in case of other tasks, with learning the efficient co-activation, it is practically regulating and controlling the mechanical impedance of the limb.

Regarding the importance of mechanical impedance [7-10] and incapability of the conventional methods to measure it, this research aims to develop a novel method for measuring human arm's mechanical impedance which is usable for the assessment of motor function of patients undergoing rehabilitation.

To achieve this goal a compliance-adaptive apparatus for upper limb rehabilitation is developed. A 4-bar linkages mechanism is designed and fabricated as the upper limb rehabilitation apparatus. The 4-bar linkages mechanism is able to provide a set of motions for the patients to exercise their upper limb. Also a sensor-less method (using transduction matrix of the motor) is used for assessing the condition of the patient's arm.

\section{DESIGN OF THE REHABILITATION APPARATUS}

\section{A. 3D Design and Fabrication}

Figure 1 shows the initial design of the 4 bar linkages mechanism using SAM software. The initial design was just a simple 4-bar linkages design, where link 1 is fixed to the ground as shown. Link 2 is the driving link that was turned by 
the DC motor. This initial design is purposely simple so that it can be delivered to clinics with an affordable price.

The design was then change into the one shown in Figure 2 and Figure 3. In this design, the link 4 of the previous design was changed into a coupler. The idea was to design a set of couplers which can be detached and fixed to joint 3 and 4 of the 4-bar linkages mechanism to achieve different motion paths. Each coupler on the plexiglass disc is able to produce a motion trajectory. The coupler was designed in form of a big disc with a number of holes drilled around it to represent the coupler points. Figure 2 show a partial designed of the 4-bar linkages mechanism using Solidworks and Figure 3 and Figure 4 show the complete fabricated 4-bar linkage mechanism.

It can see in Figures 4 that a handle is used for the user to grip on while the 4 bar linkages mechanism is turning, the handle can be detached and positioned at other holes for different motion paths. A roller is used to supporting the driven link. And a direct current motor is used to turn the driving link.

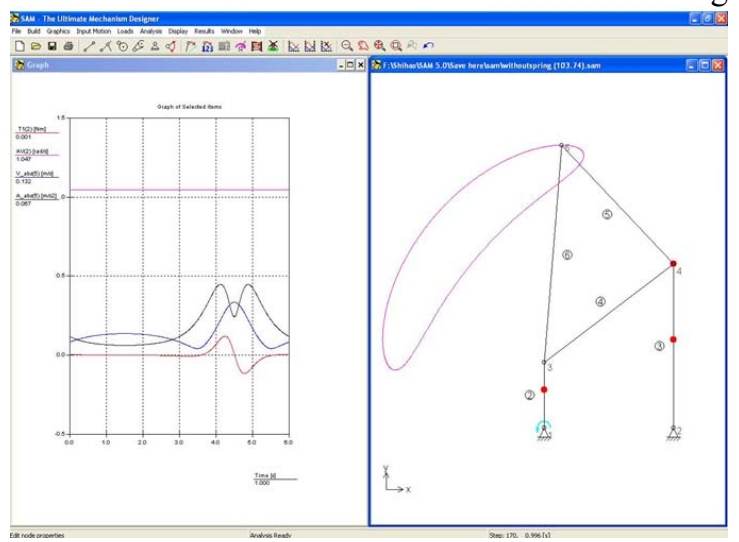

Fig. 1. Screen shot of a mechanism design session

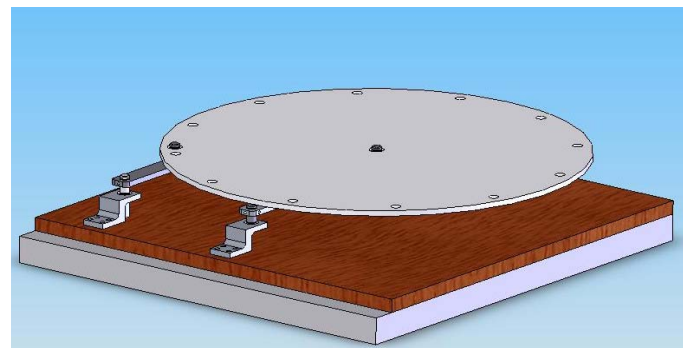

Fig. 2. design of the 4 bar linkages mechanism

\section{B. The Rehabilitation Apparatus}

The rehabilitation apparatus is developed to provide continuous motion exercises for the patient's limbs. The objectives are to prevent the patient's joints from becoming stiff due to the lack of exercising and to strengthen their upper limbs muscles. The motor was used as an actuator for turning the 4-bar linkage mechanism as well as a sensor for assessing the condition of the patient's limbs. When the patient tries to apply force to resist the motion of 4-bar linkages mechanism, the motor will experience a larger resistance force, hence a corresponding change in the output impedance value.

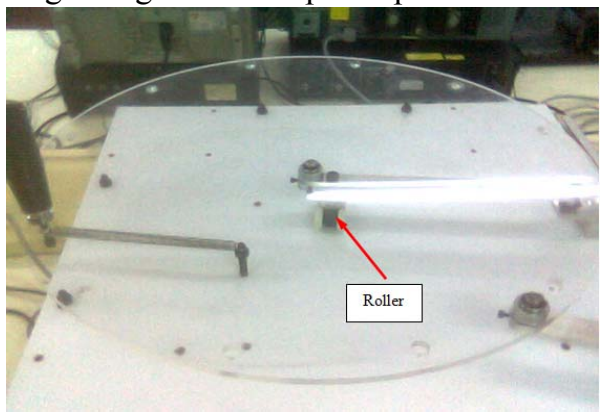

Fig. 3. mechanism after fabrication and assembly

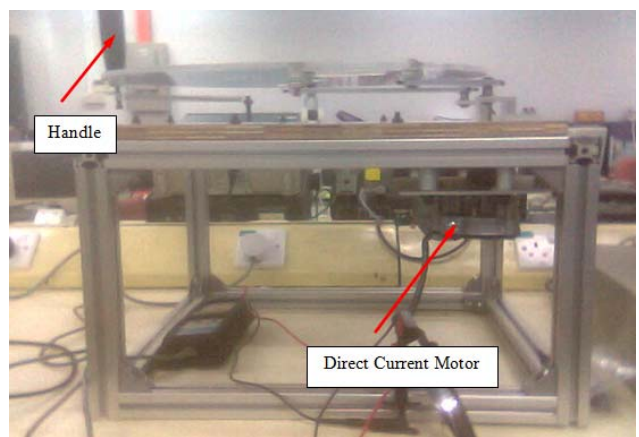

Fig. 4. DC motor (sensor cum actuator) shown below the mechanism and handle shown on top of the mechanism

Figure 5 show the how the rehabilitation apparatus is modeled. From the figure it can be seen that the electrical impedance, $Z_{\mathrm{e}}$, at the input port of the motor can be obtained by using Equation 1. And the output mechanical impedance, $\mathrm{Z}_{\mathrm{m}}$, at the output port of the 4-bar linkage mechanism can be obtained by Equation 2 .

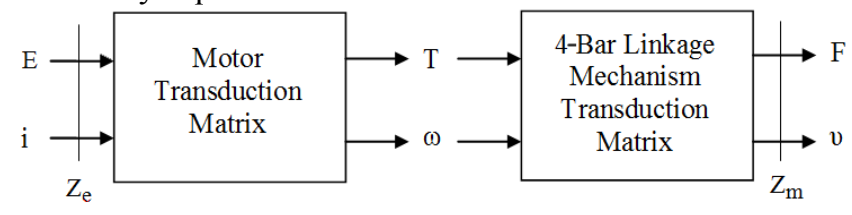

Fig. 5. Transduction Matrix of the motor and mechanism

$$
\begin{gathered}
Z_{e}=\frac{E}{i} \\
Z_{m}=\frac{F}{v}
\end{gathered}
$$

The output mechanical impedance at the output port of the 4-bar linkage mechanism is actually the resistance force (output impedance) applied by the patient's limbs.

\section{Kinematic and Dynamic Analysis Using SAM 5.0}

Before proceeding to fabrication of the 4-bar linkage mechanism, the software SAM 5.0 (Simulation and Analysis 
of Mechanisms version 5.0) was used to analyze the mechanism. Figure 1 show a screen shot of a mechanism design session. In the right side of the screen in Figure 1 a 4 bar linkage mechanism with a coupler is shown. In SAM, links are represented by lines with a node at each end, the triangle shapes are used to represent fixed joint to the ground. The crescent-like shape which is in contact with node 5 is the path on which node 5 moves. The red dot at link 2, 3 and 4 is the location of the centre of mass of the link. Other properties of link that can be input into the program are the mass and the inertia of the link. The turquoise color arrow at node 1 represents the direction of the driving link 1, the type of motion and speed of driving link can also be input into the program. Left screen of Figure 1 shows the type of analysis that is obtained from this program. SAM can be used to perform motion and force analysis of the 4-bar linkages mechanism. The mathematical foundation of the analysis kernel is finite element approach.

\section{OBTAINING THE TRANSDUCTION MATRIX}

\section{A. Transduction Matrix of the DC Motor}

Transduction Matrix (TM )as its name suggests shows the transformation between electrical input and mechanical output of an electromagnetic actuator i.e. a DC motor. As Equation 3 shows there is a relation between mechanical and electrical signals of the DC motor.

$$
\left\{\begin{array}{l}
E \\
I
\end{array}\right\}=\left[\begin{array}{ll}
T_{11} & T_{12} \\
T_{21} & T_{22}
\end{array}\right] \times\left\{\begin{array}{l}
T \\
\omega
\end{array}\right\}
$$

In this section an experimental procedure is performed which consists of a set of readings in different conditions. The reading are taken from the voltage and current signals of the motor and while simultaneously torque and angular velocity are read as well. By measuring all of the 4 variables while the motor is subjected to changes in load, the Transduction Matrix can be calibrated using least squares method.

$$
\left[\begin{array}{ccc}
E_{1} & \cdots & E_{n} \\
I_{1} & \cdots & I_{n}
\end{array}\right]=\left[\begin{array}{ll}
T_{11} & T_{12} \\
T_{21} & T_{22}
\end{array}\right] \times\left[\begin{array}{ccc}
T_{1} & \cdots & T_{n} \\
\omega_{1} & \cdots & \omega_{n}
\end{array}\right]
$$

Once the TM is obtained, the mechanical signals can be obtained from measured electrical signals according to Equation 4.

$$
\left\{\begin{array}{l}
T \\
\omega
\end{array}\right\}=\left[\begin{array}{ll}
T_{11} & T_{12} \\
T_{21} & T_{22}
\end{array}\right]^{-1} \times\left\{\begin{array}{l}
E \\
I
\end{array}\right\}
$$

Figure 6 shows the experimental setup that is used to determine the TM of the motor. In this experiment a DC power supply is used to provide power to the motor while a voltage probe, a current probe, torque meter, and tachometer are used to measure the input voltage, input current, output torque and output angular velocity of the motor respectively. These signals will then be fed into the dynamic signal analyzer for processing and analyzing purposes. Weights (lumped mass) are used to increasing the load applied to the motor. Changing the loads and recording data, will provide the elements to log into the non-square matrices of Equation 4. Using Matlab the TM was computed based on 10 different loads. The transduction matrix is shown in Equation 6.

$$
\left[\begin{array}{ll}
T_{11} & T_{12} \\
T_{21} & T_{22}
\end{array}\right]=\left[\begin{array}{ll}
0.5831 & 4.25 \\
0.3063 & 0.06
\end{array}\right]
$$

In order to validate the transduction matrix obtained above, a second set of readings were obtained from a set of second tests. Then the new data are used with the transduction matrix to compute torque and angular velocity from voltage and current. Figure 7 top shows the comparison between the measured torque and the calculated torque and Figure 7 bottom shows the comparison between the measured angular velocity and calculated angular velocity respectively.

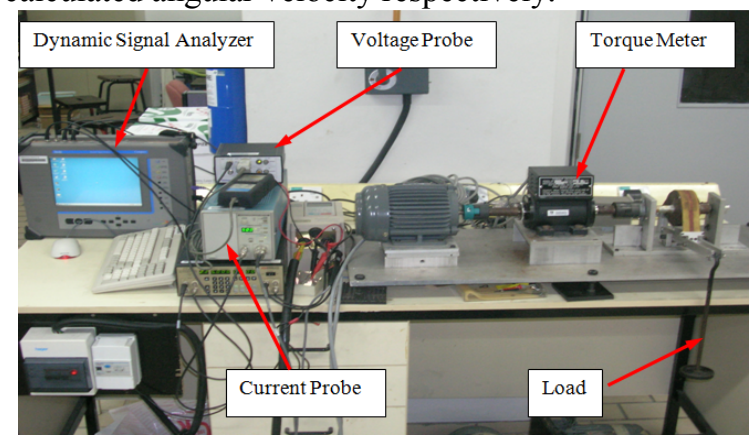

Fig. 6. Experimental setup for determining motor transduction matrix.

Overall, the discrepancies between the calculated and measured values are negligible therefore the transduction matrix obtained will be used for the computation of torque and angular velocity from the voltage and current.

\section{B. Transformation Matrix of the 4-Bar Linkage Mechanism}

To be able to obtain the force and velocity at the handle where the subject interact with the mechanism from the electrical signals of the motor, we also need to obtain the transformation matrix of the mechanism itself. From these 20 set of simulations in SAM 5.0, the matrix of the 4-bar linkage mechanism for each degree of position $(\theta)$ of the driving link can be obtained using equation 7 .

As Equation 7 shows, the transformation matrix transforms the torque and angular velocity to force and velocity. To obtain the matrix least squares approximation is used. Figures 8 show the elements of the transformation matrix with respect to the angular position $\theta$. Note that in Figure $83^{\text {rd }}$ panel from top, which shows the values of $\mathrm{A}_{21}$, the numbers are nearly zero.

$$
\left[\begin{array}{lll}
T_{1} & \cdots & T_{n} \\
\omega_{1} & \cdots & \omega_{n}
\end{array}\right]=\left[\begin{array}{ll}
A_{11} & A_{12} \\
A_{21} & A_{22}
\end{array}\right] \times\left[\begin{array}{ccc}
F_{1} & \cdots & F_{n} \\
v_{1} & \cdots & v_{n}
\end{array}\right]
$$



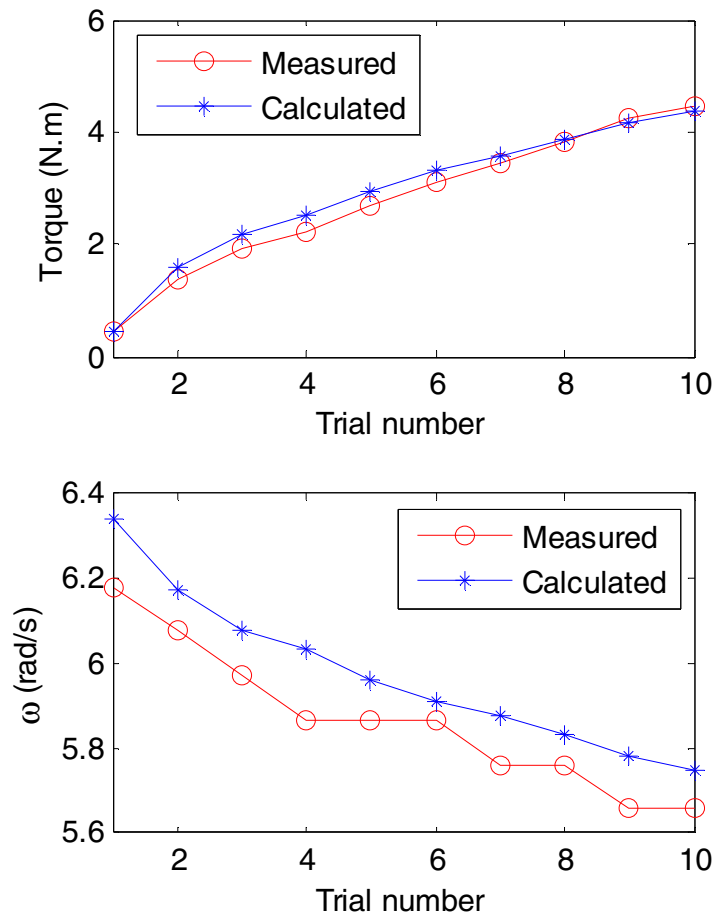

Fig. 7. Measured vs. calculated values of torque.

The transformation matrix is then used to obtain the force and velocity from torque and angular velocity according to Equation 8.

$$
\left\{\begin{array}{l}
F \\
v
\end{array}\right\}=\left[\begin{array}{ll}
A_{11} & A_{12} \\
A_{21} & A_{22}
\end{array}\right]^{-1}\left\{\begin{array}{l}
T \\
\omega
\end{array}\right\}
$$

Finally, the force and velocity can be computed based on voltage and current of the motor as shown in Equation 9.

$$
\left\{\begin{array}{l}
F \\
v
\end{array}\right\}=\left[\begin{array}{ll}
A_{11} & A_{12} \\
A_{21} & A_{22}
\end{array}\right]^{-1} \times\left[\begin{array}{ll}
T_{11} & T_{12} \\
T_{21} & T_{22}
\end{array}\right]^{-1} \times\left\{\begin{array}{c}
E \\
I
\end{array}\right\}
$$

When the mechanism is run by itself without an external load, the impedance is obtained by both theoretical and experimental procedure. For the theoretical one, the value of impedance is obtained using Equation 2, by dividing force over velocity both of which simulated in SAM 5.0.

For the experimental measurement of the mechanical impedance, the measurement of electrical voltage and current led to the use of the transduction matrix to obtain torque and angular velocity which are then inputted to the transformation matrix of the 4-bar linkage mechanism to obtain force and velocity. Finally, force and velocity are introduced to Equation 2 to obtain the mechanical impedance.

Figure 9 compares the simulated mechanical impedance to the mechanical impedance obtained from the measurements. Both graphs are plotted against the angular position $\theta$. As can be seen, there is a very good agreement between the two graphs (theoretical and experimental). This accuracy supports the use of the system together with the measurement method for measuring the impedance of human arm that may be added to the system when a human subject holds the handle of the 4-bar linkage during motion.
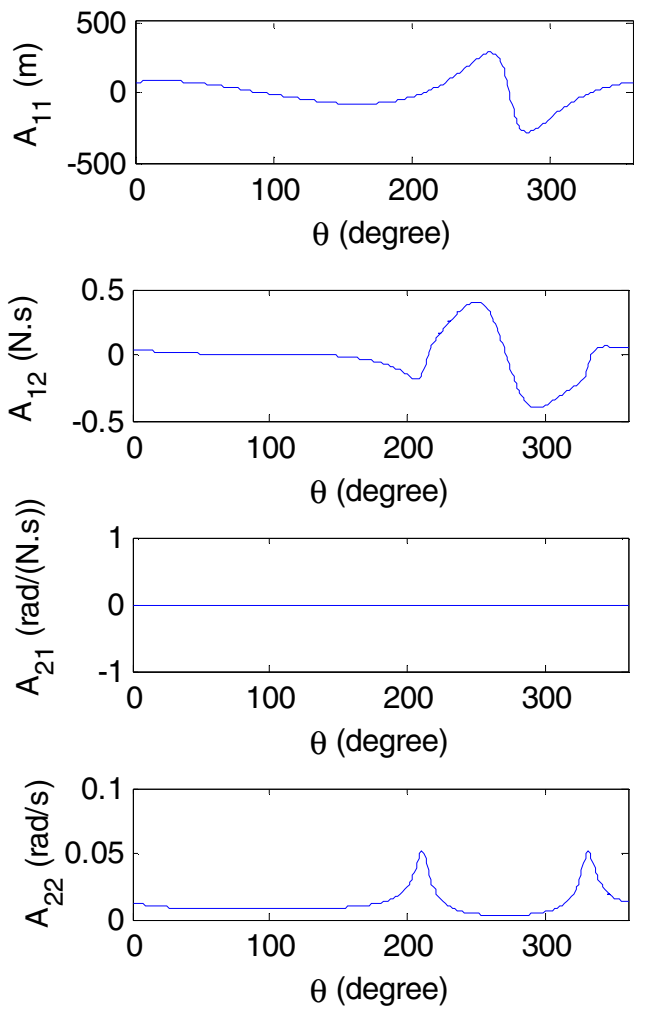

Fig. 8. $\mathrm{A}_{11}, \mathrm{~A}_{12}, \mathrm{~A}_{21}, \mathrm{~A}_{22}$ versus $\theta$ (x-axes)

\section{DISCUSSION AND COLNCLUSION}

In this paper, an upper limb rehabilitation apparatus was designed and developed to create different continuous motion trajectories through the turning of 4 bar linkages mechanism by a direct current motor. The transduction matrix of the motor was then obtained experimentally by measuring inputs (voltage and current) signals and the output (torque and angular velocity) signals. The concept of using the input electrical impedance for monitoring the output mechanical impedance was also proven to work for the obtained transduction matrix in one of the experiments. Overall, the idea of using the motor as a sensor cum actuator for assessing the strength of the user hand proved feasible.

The comprehensive future goal of this work is to be part of a wider scope of affordable [11], [12] home-based robotic [13], [14] and non-robotic rehabilitation [15] setting for monitoring [16] and training [17], [18]of stroke patients.

\section{ACKNOWLEDGMENT}

This research was done in Mechanics of Machine Lab in the School of Mechanical and Aerospace Engineering, 
Nanyang Technological University. The technical assistance of the lab staff and student lab mates was a great help.
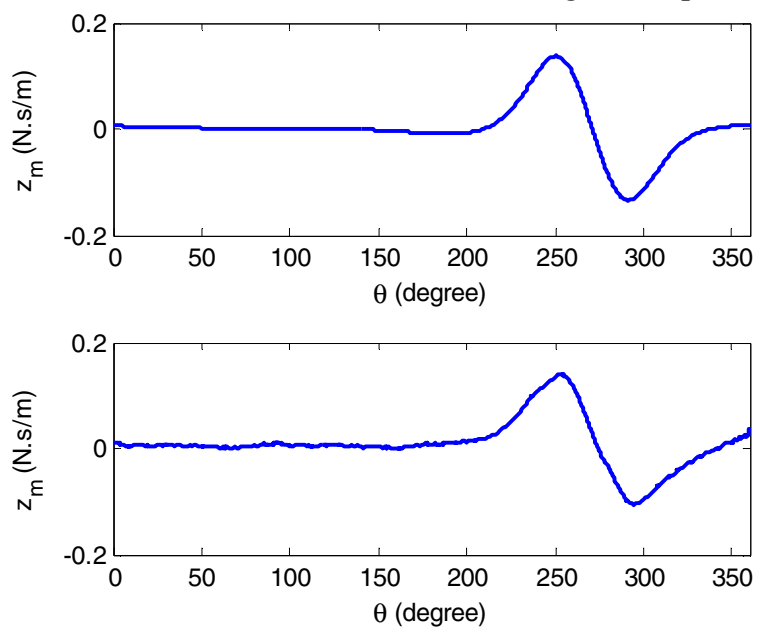

Fig. 9. Mechanical impedance obtained from SAM 5.0 simulation (top panel) versus the impedance obtained from the experimental measurements (bottom panel)

\section{REFERENCES}

[1] Fugl-Meyer AR, Jääskö L, Leyman I, Olsson S, and Steglind S, "The post-stroke hemiplegic patient. 1. a method for evaluation of physical performance.," Scandinavian Journal of Rehabilitation Medicine, vol. 7, no. 1, pp. 13-31, 1975.

[2] J. J. Palazzolo, M. Ferraro, H. I. Krebs, D. Lynch, B. T. Volpe, and N. Hogan, "Stochastic Estimation of Arm Mechanical Impedance During Robotic Stroke Rehabilitation," IEEE Transactions on Neural Systems and Rehabilitation Engineering, vol. 15, no. 1, pp. 94 103, Mar. 2007.

[3] N. Hogan, "Adaptive control of mechanical impedance by coactivation of antagonist muscles," IEEE Transactions on Automatic Control, vol. 29, no. 8, pp. 681 - 690, Aug. 1984.

[4] H. M. Hondori, L. S. Fu, and R. Khosrowabadi, "Muscles Co-activation in a Stationary Limb Altered According to Movement of Other Limb," presented at the BIODEVICES 2010, 2010, pp. 163-165.

[5] E. Burdet, R. Osu, D. W. Franklin, T. E. Milner, and M. Kawato, "The central nervous system stabilizes unstable dynamics by learning optimal impedance," Nature, vol. 414, no. 6862, pp. 446-449, Nov. 2001.

[6] M. Darainy and D. Ostry, "Muscle cocontraction following dynamics learning," Experimental Brain Research, vol. 190, no. 2, pp. 153-163, 2008.

[7] Hossein Mousavi Hondori, "Measuring Human Arm's Mechanical Impedance for Assessment of Motor Function," Nanyang Technological University, Singapore, 2012.
[8] H. M. Hondori and L. Shih-Fu, "A method for measuring human arm's mechanical impedance for assessment of motor rehabilitation," in Proceedings of the 3rd International Convention on Rehabilitation Engineering \& Assistive Technology, New York, NY, USA, 2009, pp. $17: 1-17: 4$.

[9] H. M. Hondori and S. F. Ling, "A Novel Device for Measuring Mechanical Impedance during Dynamic Tasks," presented at the BIODEVICES 2010, 2010, pp. 64-68.

[10] H. M. Hondori and L. Shih-Fu, "Perturbation-based measurement of real and imaginary parts of human arm's mechanical impedance," in 2010 Annual International Conference of the IEEE Engineering in Medicine and Biology Society (EMBC), 2010, pp. 5911-5914.

[11] H. M. Hondori and A. W. Tech, "Smart mug to measure hand's geometrical mechanical impedance," in 2011 Annual International Conference of the IEEE Engineering in Medicine and Biology Society,EMBC, 2011, pp. $4053-4056$.

[12] Hossein Mousavi Hondori, Maryam Khademi, and Cristina Videira Lopes, "Use of a Portable Device for Measuring Arm's Planar Mechanical Impedance during Motion," in 2012 IEEE EMBS Conference on Biomedical Engineering and Sciences (IECBES).

[13] M. Khademi, H. M. Hondori, Cristina Videira Lopes, Lucy Dodakian, and Steve C. Cramer, "Haptic Augmented Reality to Monitor Human Arm's Stiffness in Rehabilitation," in 2012 IEEE EMBS Conference on Biomedical Engineering and Sciences (IECBES), 2012.

[14] H. M. Hondori, A. W. Tech, M. Khademi, and C. V. Lopes, "Real-time Measurement of Arm's Mechanical Impedance with Augmented Reality Illustration," 2012.

[15] H. M. Hondori, M. Khademi, L. Dodakian, S. C. Cramer, and C. V. Lopes, "A Spatial Augmented Reality Rehab System for Post-Stroke Hand Rehabilitation," presented at the 2013 Conference on Medicine Meets Virtual Reality, NextMed/MMVR20, 2013.

[16] H. M. Hondori, M. Khademi, and C. V. Lopes, "Monitoring Intake Gestures using Sensor Fusion (Microsoft Kinect and Inertial Sensors) for Smart Home Tele-Rehab Setting," in 2012 1st Annual IEEE Healthcare Innovation Conference, Houston, TX, 2012.

[17] H. M. Hondori and L. Shih-Fu, "Monitoring Human Arm's Mechanical Impedance while Adapting a Reaching Task."

[18] H. M. Hondori and L. S. Fu, "A Simultaneous Sensing cum Actuating Method for Measuring Human Arm's Mechanical Impedance." 